\title{
Brain metastases from breast cancer may respond to endocrine therapy: report of two cases
}

This article was published in the following Dove Medical Press journal:

OncoTargets and Therapy

\author{
Qian Wang* \\ Bing Sun* \\ Chao Liu \\ Sanzhong Shi \\ Lijuan Ding \\ Jiannan Liu \\ Shikai Wu
}

Department of Radiation Oncology, Affiliated Hospital of Academy of Military Medical Sciences, Beijing, China

*These authors contributed equally to this work
Correspondence: Shikai Wu Department of Radiation Oncology, Affiliated Hospital of Academy of Military Medical Sciences, No 8 East Road, Fengtai District, Beijing 10007I, China

Tel +86 I58 I0037307

Email skywu4923@sina.com

\begin{abstract}
Brain metastases from breast cancer have a poor prognosis. There have been few cases reported where patients with breast cancer and brain metastases respond well to endocrine therapy (tamoxifen or letrozole). Here, we report the cases of two breast cancer patients with brain metastases who responded to medroxyprogesterone acetate and fulvestrant, respectively. These cases indicate that endocrine therapy could be very effective in the management of brain metastases from breast carcinoma.
\end{abstract}

Keywords: breast cancer, brain metastases, endocrine therapy, medroxyprogesterone acetate, fulvestrant

\section{Introduction}

Breast cancer is the second most common cause of brain metastasis (BM) among all solid cancers, with metastases occurring in $10 \%-16 \%$ of patients with breast cancer and in as high as $30 \%$ of autopsy studies. ${ }^{1}$ It is widely reported that patients with breast cancer-related BM usually have a poor prognosis and their survival in the absence of any form of treatment is $<2$ months. ${ }^{2}$ Survival rates after BM were related with the subtype of the primary tumor. Human epidermal growth factor-2 (HER-2)-positive patients have a significantly better prognosis compared with other subtypes, and triplenegative patients have the worst prognosis. ${ }^{3,4}$

The various ways of treating of BM include surgery, radiotherapy (whole-brain radiotherapy and stereotactic radiosurgery), and systemic therapy. Corticosteroids and local treatments, including radiotherapy and neurosurgical resection, remain the cornerstones of therapy for BM. The effectiveness of chemotherapeutics in patients who received prior or concurrent cranial radiotherapy is reported with response rates of $50 \%-59 \% .^{5}$ Even poorer is the actual response to chemotherapy alone, which is dismal and lacks research on the response of monotherapy. The poor response might be due to the limited ability of the tumor to cross the blood-brain barrier $(\mathrm{BBB})$ and a relatively chemoresistant stage after receiving multi-line treatment. ${ }^{2}$ Therefore, the prognosis for the majority of patients with BM remains poor, despite recent advances in local and systemic therapies, with a median survival of around 10 months. $^{2}$

Intriguingly, there have been few cases reported where patients with breast cancer $\mathrm{BM}(\mathrm{BCBM})$ have responded well to endocrine monotherapy. ${ }^{6-14}$ In fact, all reported cases of success happened before the year 2000, and the hormonal therapy with a long-term remission of BMs only included treatment with tamoxifen, letrozole, and megestrol acetate. In this report, we present two patients with BCBM who reacted to two different forms of endocrine therapy; one patient responded well to 
medroxyprogesterone acetate (MPA) and the other patient achieved a temporary partial response (PR) to fulvestrant.

\section{Case report Case I}

In January 2007, a 38-year-old Chinese female diagnosed with right breast cancer underwent breast-conserving surgery and axillary dissection for an invasive ductal carcinoma that was estrogen receptor (ER) positive, progesterone receptor (PR) positive, HER-2 negative, and Ki-67 (25\%-50\%), with 4 metastatic lymph nodes in the 31 dissected lymph nodes. The pathological stage was IIIA (T2N2M0). After surgery, the patient received chemotherapy, radiotherapy, and hormonal therapy (tamoxifen) as adjuvant therapy. In December 2007, she developed three nodules in the right breast, and histology showed malignant cancer cells. She refused to undergo surgery due to personal reasons and chose to start capecitabine chemotherapy instead. Following five cycles of chemotherapy, the patient was revealed to have had a complete regression of the local tumor, and she then chose to perform a rigorous observation of the local tumor. By July 2009, she had again developed a new local lump in the right breast with progressive enlargement, but she did not undergo further inspections and treatment. Later, in September 2010, the patient was referred to our hospital with lung metastases, pleural effusion, multiple bone metastases, and asymptomatic BMs. Histopathologic examination of the lump in the right breast indicated that the tumor was ER positive, PR positive, and HER-2 negative. Magnetic resonance imaging (MRI) of the lump showed four hyperdense parenchymal metastatic lesions in the frontal, parietal, and cerebellum regions, with a diameter of $3-8 \mathrm{~mm}$, along with a right frontal bone lesion and suspicious adjacent meningeal metastasis (Figure 1). After a comprehensive consideration of treatment options and her poor performance status, we decided not to perform further cerebrospinal fluid (CSF) examination and chemoradiotherapy. She was treated daily with $1,000 \mathrm{mg}$ of MPA from September 27, 2010 through November 23, 2010, the day she achieved a confirmed PR against the extracranial disease, to August 2011, when a follow-up of an MRI imaging of the brain showed a complete resolution of the brain parenchyma lesions (Figure 1B and D). The field of suspicious meningeal metastasis did not change significantly on the MRI scan, and the patient still did not have any meningeal metastasis-related symptoms. However, the lung metastases and local tumors of the right breast progressed, and then she stopped taking MPA. Subsequently, she was switched over to letrozole and goserelin acetate in August 2011, and this regimen showed a PR with a progression-free
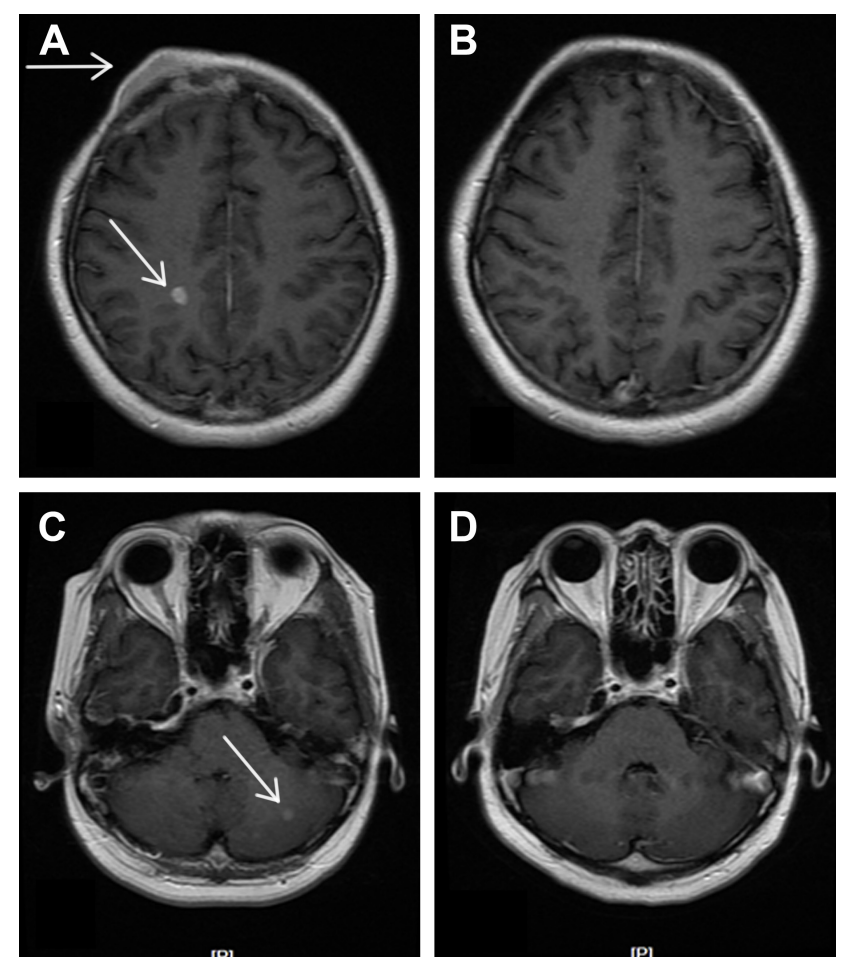

Figure I Enhanced MRI of a $6 \mathrm{~mm}$ slice at the start of the treatment with MPA showing multiple brain metastases $(\mathbf{A}, \mathbf{C})$ vs a repeat MRI of the same patient showing complete resolution of the metastatic lesions of the brain 10 months after MPA treatment (B, D). The arrows in images $\mathbf{A}$ and $\mathbf{C}$ indicated the metastatic tumors. Abbreviations: MPA, medroxyprogesterone acetate; MRI, magnetic resonance imaging.

survival of 23 months. Regretfully, we could not evaluate the reaction of BMs to treatment further because the patient would not undergo a radiology examination of the brain during her follow-up. Nevertheless, that the patient did not exhibit any neurological symptoms during follow-up was an encouraging finding.

\section{Case 2}

A 37-year-old woman was diagnosed with an invasive ductal right breast cancer (the clinical stage was IIIC, T2N3M0) in August 2016. The tumor was ER positive, PR negative, HER-2 negative, and Ki-67 (30\%). She was treated with three cycles of anthracycline combined with cyclophosphamide. In October 2016, the patient was referred to our hospital with new right metastatic cervical lymph nodes confirmed by fine-needle aspiration. Consequently, we switched the patient to four cycles of salvage chemotherapy (vinorelbine combined with capecitabine). In February 2017, a positron emission tomography-computed tomography examination revealed metastases of the right supraclavicular and infraclavicular lymph nodes, right axillary lymph nodes, and multiple bones, as well as the presence of suspicious hilus pulmonis, mediastinum lymph 
nodes, and multiple pulmonary nodules. Considering her poor response to chemotherapy, she was subjected to an aggressive mastectomy. Pathology showed that this tumor was an invasive ductal cancer (grade III), invading the lymphovascular space and nerve and 11 metastatic lymph nodes in 13 dissected axillary lymph nodes. The patient then underwent hormonal therapy (anastrozole and bilateral ovariectomy) and concurrent radiotherapy. Immunohistochemical analysis showed that the tumor was ER positive $(>75 \%)$, PR positive ( $1 \%)$, HER-2 negative, and Ki-67 (80\%). In July 2017, the increased pulmonary nodes on computed tomography (CT) scans confirmed that lung metastases had progressed. The subsequent switch to exemestane saw a further progression of the lung metastases after 4 months. After two successive cycles of VP-16, on January 29, 2018, the patient was found to have developed an isolated BM. Brain MRI revealed a metastatic lesion, with a maximal diameter of $7 \mathrm{~mm}$, in the left parietal lobe (Figure 2). The patient was then treated with fulvestrant $500 \mathrm{mg}$ delivered by intramuscular injection, and a stereotactic radiosurgery for the control of intracranial disease was planned. We found an obvious reduction in the BM through the positioning MRI (1 month after the first dose
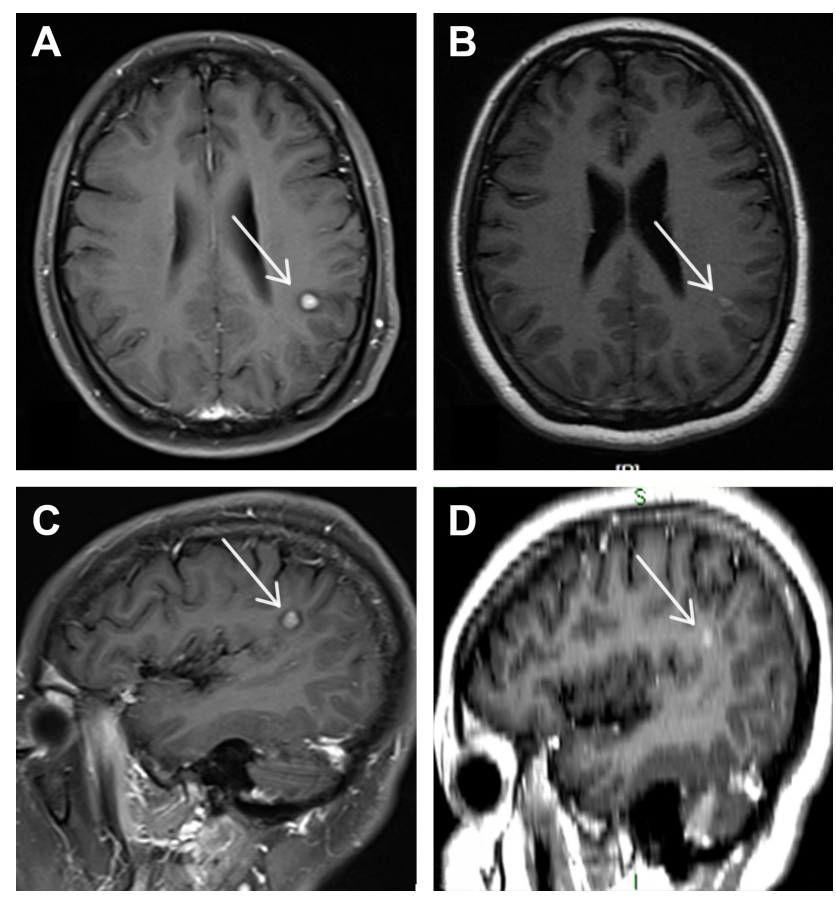

Figure 2 Enhanced MRI of a $6 \mathrm{~mm}$ slice at the start of the treatment with fulvestrant showing a single metastatic lesion on the left parietal lobe $(\mathbf{A}, \mathbf{C})$ vs a repeat MRI of the same patient showing a gross reduction in the size of the metastatic lesion of the brain I month after administering the first dose of fulvestrant (B, D).

Notes: (B) is from a positioning MRI of a $3 \mathrm{~mm}$ slice, and (D) is from three dimensional reconstruction MRI images of a $3 \mathrm{~mm}$ slice. The arrows in images $\mathbf{A}$ and C indicated the metastatic tumors.

Abbreviation: MRI, magnetic resonance imaging. of fulvestrant injection; Figure 2B and D) after treatment with fulvestrant. At the same time, the extracranial metastases did not respond much. The planned stereotactic radiotherapy (18 Gy/1 f), for the intracranial tumors, was performed and a second dose of fulvestrant was administered to the patient. The MRI showed a diminished lesion after treatment, but a new metastatic lesion in the frontal lobe appeared on March 29, 2018, 1 month after treatment with two doses of fulvestrant. The extracranial disease progressed with new liver lesions and enlarged lung lesions, features asymptomatic of an intracranial disease.

\section{Ethics statement}

The publication of this case series was approved by the Clinical Research Ethics Committee of the Affiliated Hospital of Academy of Military Medical Sciences, China (approval number: ky-2018-10-91). Written informed consent was obtained from the patients and their relatives for publication of this case series including accompanying images.

\section{Discussion}

Generally, BM in breast cancer is associated with a poor prognosis. ${ }^{15}$ The outcome of patients with BCBM remains poor even after chemotherapy and radiotherapy. The response of BCBM to antiestrogens (particularly tamoxifen) has been reported in a few cases. ${ }^{6-14}$ Previous studies of patients with BCBM treated with hormonal therapy were all reported before 2000, and the responses, then, were evaluated by CT, which did not provide a clear manifestation of BM. Here, we report a patient who responded completely to MPA and another patient with an endocrine-resistant tumor who gained a transient reduction in BM from fulvestrant after multipleline treatments.

In general, most patients with BCBM are not suitable for hormonal therapy. The majority of these patients are characterized with rapid growing, hormone receptor-negative, and/or Her-2-positive tumors, and concurrently with visceral metastases. However, some cases with hormone-dependent tumors may benefit from hormonal treatments. Cases presented in Table 1 were rare after the year 2000, because of the vigorous development of radiological and radiation therapy technology, with hormonal therapy not regularly used thereafter. Most patients who responded to hormonal treatment had long disease-free intervals and had non-visceral involvement. The first patient that we reported who was responding well to MPA exhibited similar characteristics.

Both the intracranial and extracranial lesions responded to hormonal treatment, and the response duration time of all reported cases was around 1 year. For the first case, the 


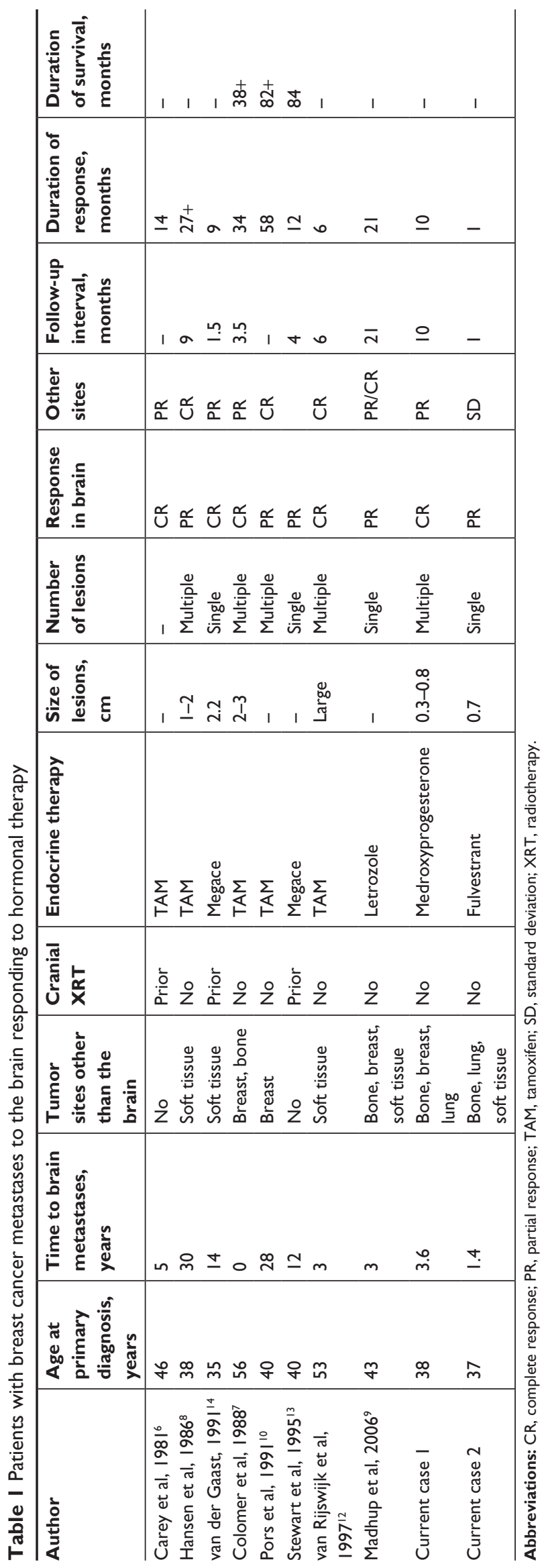

long-term control of intracranial and extracranial lesions with letrozole suggested that the patient had an estrogendependent tumor. The difference in the level of tumor regression between the lung metastases and breast tumors (PR) and the BMs (complete response) after MPA therapy could reflect the changes in ER levels among metastatic lesions or diversity in the concentration of the drug between different organs, and the nature of tumor heterogeneity. MPA has several mechanisms of action that may account for its antitumor effect in patients with metastatic tumors. MPA downregulates the expression of ER and decreases the levels of estrogen, which could inhibit tumor growth directly. A noticeable transient reduction in the sizes of BMs in case 2 suggested that fulvestrant or its metabolites entered the brain by crossing the BBB in extensive amount. The disruption of the $\mathrm{BBB}$ by metastases could have allowed the intracranial penetration of the agent. Because case 2 showed a stable response on MRI and the metastatic lesions apparently diminished (not achieved a PR response according to the Response Evaluation Criteria in Solid Tumors) after the single treatment with fulvestrant, we drew a preliminary conclusion that fulvestrant instigated the reduction in tumor size. That the intracranial tumor shrank implies a potential response of BM to fulvestrant after multiple-line treatments. The response did not last and failed to continue possibly as a result of multiple endocrine therapy-resistant mechanisms. While fulvestrant is expected to be beneficial during the management of peripheral diseases, the ability of the drug to cross the BBB is currently an uncharted territory. Abemaciclib, a CDK4/6 inhibitor, is reportedly distributed across the $\mathrm{BBB}$ and is efficient against brain metastatic tumors. ${ }^{16}$ The JPBO study (NCT 02308020) demonstrated that the unbound concentrations of abemaciclib in the plasma and tumor tissue were comparable to and consistent with the CSF concentrations of each patient and 2 of 23 BCBM patients achieved PR (8.7\%). ${ }^{17} \mathrm{~A}$ further outcome on clinical trials is expected in the near future. There are currently no case reports on BMs responding to fulvestrant, because the RT and/or chemotherapy are preferred choices of treatment for patients with $\mathrm{BCBM}$, meaning clinicians usually do not come to the conclusion we established.

There were conflicting follow-up intervals of BM from breast cancer in the reported cases, and the range of interval was from 3.5 to 18 months (Table 1). In our first case, the follow-up MRI image was performed 10 months after oral endocrine therapy and complete remission of BM was demonstrated. Outstandingly, the reduction of BM in our second case was occasionally found on the positioning MRI 
after only 1 month of administering the first dose of fulvestrant. It is, hence, suggested that more frequent follow-up imaging is vital to assess periodic and accurate responses. Another suggestion is that, to have an indication of progression, local treatment should be added immediately when BMs appear. At least, an early image assessment, 1-2 months after treatment, should be mandatory.

Lien et $\mathrm{l}^{18}$ reported that the concentration of tamoxifen and its metabolites was up to 46-fold higher in brain tissues and BMs compared with the concentration in serum. The high levels of the drug and its metabolites in metastatic tumor could be demonstrative of the association between healing agents and the receptors of tumor tissues. Antiestrogen receptors include ERs, cytochrome P-450, protein kinase $\mathrm{C}$, calmodulin, histamine-like receptors, muscarinic receptors, and dopamine receptors. Tamoxifen and its metabolites may bind to these receptors, but may also partition into the myelin layer of the brain. ${ }^{18}$ However, there is no such data available to show for MPA or fulvestrant so far. Despite the promising findings, our study requires more in-depth studies.

\section{Conclusion}

The two reported cases suggest that hormone therapy could play a role in the single therapy of selected cases of BMs from patients with disseminated breast cancer who had nonrapidly life-threatening BMs and ER-positive or hormonedependent carcinoma.

\section{Acknowledgment}

Qian Wang and Bing Sun are co-first authors for this study.

\section{Author contributions}

All authors contributed to data analysis, drafting or revising the article, gave final approval of the version to be published, and agree to be accountable for all aspects of the work.

\section{Disclosure}

The authors report no conflicts of interest in this work.

\section{References}

1. Lin NU, Amiri-Kordestani L, Palmieri D, Liewehr DJ, Steeg PS. CNS metastases in breast cancer: old challenge, new frontiers. Clin Cancer Res. 2013;19(23):6404-6418.

2. Costa R, Carneiro BA, Wainwright DA, et al. Developmental therapeutics for patients with breast cancer and central nervous system metastasis: current landscape and future perspectives. Ann Oncol. 2017;28(1): $44-56$.

3. Leone JP, Leone J, Zwenger AO, Iturbe J, Leone BA, Vallejo CT Prognostic factors and survival according to tumour subtype in women presenting with breast cancer brain metastases at initial diagnosis. Eur J Cancer. 2017;74:17-25.

4. Witzel I, Laakmann E, Weide R, et al. Treatment and outcomes of patients in the brain metastases in breast cancer network registry. Eur J Cancer. 2018;102:1-9.

5. Rostami R, Mittal S, Rostami P, Tavassoli F, Jabbari B. Brain metastasis in breast cancer: a comprehensive literature review. J Neurooncol. 2016;127(3):407-414.

6. Carey RW, Davis JM, Zervas NT. Tamoxifen-induced regression of cerebral metastases in breast carcinoma. Cancer Treat Rep. 1981; 65(9-10):793-795.

7. Colomer R, Cosos D, Del Campo JM, Boada M, Rubio D, Salvador L. Brain metastases from breast cancer may respond to endocrine therapy. Breast Cancer Res Treat. 1988;12(1):83-86.

8. Hansen SB, Galsgård H, von Eyben FE, Westergaard-Nielsen V, Wolf-Jensen J. Tamoxifen for brain metastases from breast cancer. Ann Neurol. 1986;20(4):544.

9. Madhup R, Kirti S, Bhatt ML, Srivastava PK, Srivastava M, Kumar S. Letrozole for brain and scalp metastases from breast cancer - a case report. Breast. 2006;15(3):439-441.

10. Pors H, von Eyben FE, Sørensen OS, Larsen M. Longterm remission of multiple brain metastases with tamoxifen. J Neurooncol. 1991;10(2): 173-177.

11. Salvati M, Cervoni L, Innocenzi G, Bardella L. Prolonged stabilization of multiple and single brain metastases from breast cancer with tamoxifen. Report of three cases. Tumori. 1993;79(5):359-362.

12. van Rijswijk RE, van Oostenbrugge RJ, Twijnstra A. A case of brain metastases from male breast cancer responding to tamoxifen. Eur $J$ Cancer. 1997;33(13):2282-2283.

13. Stewart DJ, Dahrouge $S$. Response of brain metastases from breast cancer to megestrol acetate: a case report. J Neurooncol. 1995;24(3):299-301.

14. van der Gaast A, Alexieva-Figusch J, Vecht C, Verweij J, Stoter G. Complete remission of a brain metastasis to third-line hormonal treatment with megestrol acetate. Am J Clin Oncol. 1990;13(6):507-509.

15. Cagney DN, Martin AM, Catalano PJ, et al. Incidence and prognosis of patients with brain metastases at diagnosis of systemic malignancy: a population-based study. Neuro Oncol. 2017;19(11):1511-1521.

16. Choo JR, Lee SC. CDK4-6 inhibitors in breast cancer: current status and future development. Expert Opin Drug Metab Toxicol. 2018; 14(11):1123-1138.

17. Kotake T, Toi M. Abemaciclib for the treatment of breast cancer. Expert Opin Pharmacother. 2018;19(5):517-524.

18. Lien EA, Wester K, Lønning PE, Solheim E, Ueland PM. Distribution of tamoxifen and metabolites into brain tissue and brain metastases in breast cancer patients. Br J Cancer. 1991;63(4):641-645.
OncoTargets and Therapy

\section{Publish your work in this journal}

OncoTargets and Therapy is an international, peer-reviewed, open access journal focusing on the pathological basis of all cancers, potential targets for therapy and treatment protocols employed to improve the management of cancer patients. The journal also focuses on the impact of management programs and new therapeutic agents and protocols on

\section{Dovepress}

patient perspectives such as quality of life, adherence and satisfaction. The manuscript management system is completely online and includes a very quick and fair peer-review system, which is all easy to use. Visit http://www.dovepress.com/testimonials.php to read real quotes from published authors. 